\title{
Pesquisa com métodos mistos na enfermagem: experiência na pós-graduação
}

Research with mixed methods in nursing: postgraduate experience

Investigación con métodos mixtos en enfermería: experiencia de posgrado

\section{João Lucas Campos de Oliveira ${ }^{\mathrm{I}}$, Ana Maria Müller de Magalhães ${ }^{\mathrm{II}}$, Juliana Aparecida Peixoto Nishiyama ${ }^{\mathrm{III}}$, Dagmar Willamowius Vituri ${ }^{\mathrm{IV}}$, Sonia Silva Marcon ${ }^{\mathrm{V}}$, Laura Misue Matsuda ${ }^{\mathrm{VI}}$}

\begin{abstract}
Resumo: Objetivo: relatar a experiência de planejamento e execução de uma pesquisa com métodos mistos na pós-graduação stricto sensu em enfermagem. Método: relato de experiência de pesquisa vinculada a Programa de Pós-Graduação em Enfermagem do Paraná e grupo de estudos na área gestão em saúde e enfermagem. O processo de planejamento e execução da investigação ocorreu no período de março de 2015 a dezembro de 2017. Resultados: o estudo foi sistematizado pelo método misto na estratégia explanatória sequencial. A experiência, descrita em três momentos/etapas: Pré-concepção, na qual realizou-se aproximação primária com a vertente metodológica; Planejamento do projeto de pesquisa, fase em que houve intensa imersão ao delineamento de estudo e objeto, culminando ao desenho de pesquisa, e; Execução, onde os procedimentos e fases da investigação planejados foram operacionalizados. Considerações Finais: apesar de desafiadora, a experiência foi profícua porque se mostrou factível à robustez dos resultados de pesquisas na enfermagem.
\end{abstract}

Descritores: Projetos de pesquisa; Metodologia; Pesquisa em enfermagem; Métodos; Enfermagem

\begin{abstract}
Objective: To report the experience of planning and executing a research with mixed methods in stricto sense postgraduate nursing. Method: report of research experience linked to the Nursing Graduate Program of Paraná and group of studies in the area of health management and nursing. The process of planning and execution of the investigation took place from March 2015 to December 2017. Results: the study was systematized
\end{abstract}

\footnotetext{
${ }^{\text {I }}$ Enfermeiro. Doutor em Enfermagem. Professor adjunto do Departamento de Assistência e Orientação Profissional da Escola de Enfermagem da Universidade Federal do Rio Grande do Sul (UFRGS). Porto Alegre/RS - Brasil. E-mail: enfjoaolcampos@yahoo.com.br ORCID iD https://orcid.org/0000-0002-1822-2360

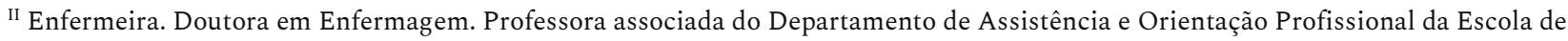
Enfermagem da Universidade Federal do Rio Grande do Sul. Porto Alegre/RS - Brasil. E-mail: amagalhaes@hcpa.edu.br ORCID iD https://orcid.org/0000-0003-0691-7306

${ }^{\text {III }}$ Enfermeira. Mestranda do Programa de Pós-Graduação em Enfermagem da Universidade Federal de Mato Grosso (UFMT). Cuiabá/MT Brasil. E-mail: julynishiyama@gmail.com ORCID ID https://orcid.org/0000-0001-5941-7351

${ }^{\text {IV }}$ Enfermeira. Doutora em Ciências. Coordenadora do Núcleo Interno de Regulação do Hospital Universitário de Londrina. Londrina/PR Brasil. E-mail: dagvituri@gmail.com ORCID ID https://orcid.org/0000-0002-7663-1736

v Enfermeira. Doutora em Filosofia da Enfermagem. Professora titular do Departamento de Enfermagem da Universidade Estadual de Maringá. Maringá/PR - Brasil. E-mail: soniasilva.marcon@gmail.com ORCID iD https://orcid.org/0000-0002-6607-362X

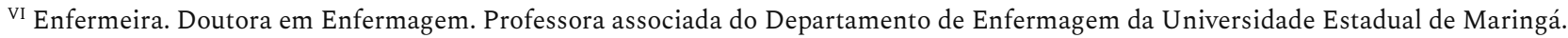
Maringá/PR - Brasil. E-mail: lauramisuem@gmail.com ORCID ID https://orcid.org/0000-0002-4280-7203
} 
by the mixed method in the sequential explanatory strategy. The experience, described in three moments / stages: Preconception, in which a primary approach was made to the methodological aspect; Research project planning, phase in which there was intense immersion in the study and object design, culminating in the research design, and; Execution, where the procedures and planned investigation phases were operationalized. Final Thoughts: Although challenging, the experience was fruitful because it proved feasible to the robustness of nursing research results.

Descriptors: Research projects; Methodology; Nursing research; Methods; Nursing

Resumen: Objetivo: Informar sobre la experiencia de una investigación con métodos mixtos en enfermería de posgrado estrictamente sentido. Método: experiencia investigadora vinculada al Programa de Posgrado en Enfermería de Paraná y grupo de estudios en el área de gestión de salud y enfermería. El proceso de planificación y ejecución de la investigación tuvo lugar de marzo de 2015 a diciembre de 2017. Resultados: el estudio fue sistematizado por el método mixto en la estrategia explicativa secuencial. La experiencia, descrita en tres momentos/etapas: Preconcepción, en la cual se realizó un enfoque primario del aspecto metodológico; Planificación de la investigación, fase en la que hubo una inmersión intensa en el estudio y el diseño del objeto, que culminó en el diseño de la investigación, y; Ejecución, donde se operacionalizaron los procedimientos planificados. Reflexiones finales: la experiencia fue fructífera porque resultó factible para la solidez de los resultados de la investigación de enfermería.

Descriptores: Proyectos de investigación; Metodología Investigación en enfermería; Métodos; Enfermería

\section{Introdução}

No mundo da pesquisa científica, o emprego de abordagens metodológicas inovadoras é profícuo não somente na busca de evidências científicas sólidas, mas também como forma de responder às exigências crescentes deste universo, expressivamente afiançado pela pósgraduação stricto sensu. Neste escopo, os métodos mistos têm sido elencados como o terceiro e inovador paradigma da pesquisa científica, com potencial para alcançar resultados robustos e inferências mais acuradas. ${ }^{1-3}$

Pesquisa de métodos mistos compreende investigação científica que combina as abordagens quantitativa e qualitativa em um mesmo estudo, utilizando-se de procedimentos próprios de ambas as vertentes, mas combinados (“mixados”) entre si de alguma forma e em algum momento do estudo. ${ }^{4-5}$ Em outras palavras, não se limita à métrica e/ou a compreensão subjetiva dos fatos, uma vez que busca integrá-las. Logo, torna-se particularmente útil para reduzir as "contradições” comuns entre achados quantitativos e qualitativos. ${ }^{6}$ 
Os estudos com métodos mistos não são pesquisas "quanti-qualitativas” ou "qualiquantitativas”. Isso porque, de acordo com estudiosos que se dedicam a delinear estratégias de investigação mista, em especial John W. Creswell, existem princípios que regem um estudo verdadeiramente misto, tais como: distribuição de tempo, atribuição de peso, combinação de dados e teorização. ${ }^{1,4-5}$

Não é pretensão deste estudo discorrer, ainda que de forma genérica, acerca dos princípios e procedimentos das pesquisas com métodos mistos, porque se vale do relato de uma experiência circunscrita, que respeitou integralmente tais princípios em um determinado estudo. Portanto, registra-se aqui algumas indicações de leitura que possam esclarecer a respeito dos princípios, procedimentos e estratégias "gerais" elementares das pesquisas mistas. ${ }^{1-}$ 5,9

$\mathrm{Na}$ pesquisa em enfermagem, a utilização dos métodos mistos tem sido referida como altamente valiosa para maior ou melhor entendimento dos fenômenos de estudo, naturalmente arraigados de densa complexidade, já que a combinação das abordagens quantitativa e qualitativa parece alcançar níveis mais elevados de compreensão dos resultados vinculados direta ou indiretamente ao cuidado humano, sua gestão, ensino e a própria pesquisa. ${ }^{2-3,8-10,13}$ Desta forma, a integração de ambas as abordagens consolida o arcabouço teórico de suporte ao estudo na área de enfermagem, e, assim, atribui maior densidade aos estudos da área, visto que o conjunto de dados gerados é mais abrangente, e as inferências das duas abordagens são interpretadas conjuntamente. ${ }^{8}$

Apesar das potencialidades voltadas principalmente pela “atenuação” das limitações de uma e/ou outra abordagem (quantitativa e qualitativa) da pesquisa de métodos mistos, uma revisão crítica da literatura recente conduzida por pesquisadores italianos atesta que há escassez de orientações mais claras para a condução de pesquisas ancoradas nos métodos mistos na 
enfermagem, e que existe a necessidade de se definir diretrizes de pesquisas robustas para que os resultados possam ser de fato transpostos para a prática profissional. ${ }^{11}$

Com base na prerrogativa anterior, acredita-se que é de interesse à área de enfermagem, inclusive para sua consolidação como ciência, a descrição de experiências exitosas acerca da utilização de métodos mistos de pesquisa, pois isso pode auxiliar pesquisadores interessados a direcionarem-se em tal vertente metodológica inovadora e recente na área, inclusive no Brasil. Ademais, sabe-se que a pós-graduação ocupa evidente posição de impacto na produção do conhecimento, o que não é diferente na enfermagem. Assim, o objetivo deste estudo consiste em relatar a experiência de planejamento e execução de uma pesquisa com métodos mistos na pósgraduação stricto sensu em enfermagem.

\section{Método}

Trata-se de um relato de experiência embasado na vivência de planejamento e execução de Projeto de Pesquisa ancorado na abordagem de métodos mistos. A pesquisa foi vinculada à pós-graduação stricto sensu em enfermagem, nível Doutorado, junto ao Programa de PósGraduação em Enfermagem da Universidade Estadual de Maringá (UEM) e vinculada ao Núcleo de Pesquisa, Prática e Ensino em Gestão em Saúde (NUPPEGES).

O período de planejamento e execução do Projeto de Pesquisa ocorreu entre março de 2015 a dezembro de 2017. No entanto, a imersão no objeto de estudo a ser relatado se deu ainda no curso de Mestrado, pelo pesquisador principal, desde 2013.

A fim de sistematizar o presente estudo, optou-se por descrevê-lo em três momentos/etapas, que emergiram da própria experiência autoral: 1) Pré-concepção do Projeto de Pesquisa com métodos mistos; 2) Planejamento do Projeto de Pesquisa com métodos mistos e; 3) Execução do Projeto do Projeto de Pesquisa com métodos mistos.

Este estudo não fere os princípios éticos que regem as pesquisas com seres humanos, pois se trata de uma experiência relatada sobre o processo de investigação eminentemente autoral, e 
5 | Oliveira JLC, Magalhães AMM, Nishiyama JAP, Vituri DW, Marcon SS, Matsuda LM

não de resultados de pesquisa. Todavia, o estudo que originou este relato foi aprovado em Comitê de Ética em Pesquisa sob o parecer número 1.788.249/2016, e está nacionalmente cadastrado sob protocolo de CAAE: 58571216.4.0000.0104.

\section{Descrição da experiência}

\section{Pré-Concepção do Projeto de Pesquisa com Métodos Mistos}

No curso de Mestrado, investigou-se a Acreditação na vertente/abordagem qualitativa de pesquisa. Os resultados deste estudo permitiram a apreciação geral do objeto, com algumas aproximações mais pontuais. Estas, além de viabilizar maior entendimento no que se refere aos diversos aspectos da Acreditação, emanaram questões ou problemas de pesquisa que poderiam ou deveriam ser respondidas. Uma das questões dizia respeito à necessidade de mensurar os possíveis - benefícios da Acreditação hospitalar, inclusive em relação a aspectos que delineiam diretamente o trabalho (processo e produto) da equipe de enfermagem. Isso porque, existia a especificidade da pós-graduação em questão (Doutorado em Enfermagem), além de características e predileções autorais.

Ante o exposto, a ideia preliminar de desenvolver o Projeto de Tese de Doutorado com base em um estudo transversal analítico, eminentemente de caráter comparativo entre instituições hospitalares (considerando a presença e ausência da certificação de Acreditação), foi criando forma, ainda que sem definição clara de qual aspecto seria eleito à mensuração pretendida. Nesse momento, o problema de pesquisa ainda não estava delineado, mas já havia clareza da necessidade de se identificar algum(ns) benefício(s) ou não da Acreditação hospitalar, por meio da métrica e do pleno respeito aos princípios da pesquisa quantitativa.

$\mathrm{Na}$ intenção de submeter à Acreditação à mensuração e com base nas vivências no NUPPEGES; nos problemas de pesquisa levantados no curso de Mestrado e de intensas e contínuas buscas de material bibliográfico nacional e internacional a respeito da Acreditação e 
Pesquisa com métodos mistos na enfermagem: experiência na pós-graduação I 6

da própria Gestão da Qualidade na Saúde e Enfermagem, optou-se por delimitar o projeto em questão pautando-o na satisfação profissional da equipe de enfermagem e na satisfação de pacientes/clientes com o cuidado de enfermagem, no escopo da Acreditação hospitalar.

Frente à ideia preliminar mencionada, ao contatar a possível orientadora de Doutorado a respeito do pré-projeto enunciado, acenou-se a primeira perspectiva/sugestão de desenvolver um estudo de métodos mistos, uma vez que a pesquisadora havia participado recentemente de evento sediado na Escola de Enfermagem de Ribeirão Preto da Universidade de São Paulo (EERP-USP), onde teve seu primeiro contato com a abordagem e suas, a priori, vastas potencialidades. Desta forma, por meio de leituras preliminares sobre métodos mistos, o pesquisador percebeu a profundidade e inovação metodológica deste novo paradigma científico, e, por abarcar sua experiência qualitativa prévia em conjunto à necessidade de mensurar "efeitos" da Acreditação, a pesquisa de métodos mistos, pareceu, a princípio, ideal.

Ante o exposto, o pesquisador candidato ao curso de Doutorado reformulou o pré-projeto - ainda em âmbito individual - agregando algumas tímidas intenções de delineamento da pesquisa mista no estudo, sem tempo hábil para a devida imersão teórica a respeito da vertente metodológica.

Com a aprovação e ingresso ao curso de Doutorado, a - ainda no campo das ideias intenção de desdobrar o Projeto de Tese foi sedimentando-se, pois, o pesquisador iniciou imersão profunda ao estudo dos métodos mistos. Isso incluiu indicações de leituras por uma terceira pesquisadora mais experiente na área, sediada na Escola de Enfermagem da Universidade Federal do Rio Grande do Sul.

O contato com a pesquisadora referida se deu via e-mail, pois, ao que se conhecia da literatura na enfermagem, e, precisamente na área de gerenciamento dos serviços de enfermagem, a pesquisadora despontava como autora pioneira na pesquisa mista nestes 
escopos. Entre outras, apontou com maior ênfase as obras de John W. Creswell, fato que culminou ao início da etapa de planejamento do estudo misto.

\section{Planejamento do Projeto de Pesquisa com Métodos Mistos}

A etapa de planejamento do estudo com métodos mistos iniciou com ampla dedicação à apreensão da obra de Creswell e, assim, instrumentalização do pesquisador sobre os procedimentos técnicos de pesquisa, sem, no entanto, deixar de dar seguimento à contínua apropriação do objeto de estudo, a Acreditação hospitalar. Este processo foi intenso, teve duração de aproximadamente um ano e culminou em uma publicação de teor "didático” à pesquisa mista em enfermagem. ${ }^{3}$

Ante às leituras realizadas e, principalmente, em respeito a pré-concepção do Projeto de Pesquisa com ênfase na mensuração de possíveis efeitos da Acreditação no trabalho da enfermagem, percebeu-se a convergência na escolha por um desenho/estratégia de pesquisa mista entre aqueles descritos por Creswell. Isso porque, a estratégia explanatória sequencial, que tem atribuição de peso quantitativa (QUAN) sobre a qualitativa (qual) é altamente valiosa para aprofundar (qualitativamente) dados estatísticos primários. ${ }^{14-5}$ Logo, respeitando as ideias preliminares do estudo misto, evidenciou-se seu caráter de maior peso à abordagem quantitativa, contudo, incutido à abordagem mista de pesquisa por meio de procedimento de conexão de dados QUAN e qual.

Apesar da clareza na definição da estratégia de pesquisa mista, percebeu-se que, segundo Creswell, tal como em outras modalidades de estudos mistos sequenciais, uma das maiores dificuldades da estratégia explanatória sequencial é o provável longo período de coleta de dados. ${ }^{4}$ Assim, na etapa de planejamento da pesquisa, percebeu-se que não seria viável investigar a satisfação de pacientes com o cuidado de enfermagem juntamente com a satisfação de trabalhadores, pois a estratégia eleita demanda rigoroso respeito aos princípios da pesquisa 
quantitativa, entre eles, a amostragem representativa, o que, por se tratar de uma pesquisa sem financiamento, "individual" e com prazo limitado de execução, isto seria uma barreira importante à condução do estudo.

Frente ao exposto, optou-se por eleger outro indicador ou métrica voltado ao próprio trabalho de enfermagem, além da satisfação profissional já definida, como fator de análise em relação à Acreditação hospitalar. Destarte, emergiu o ambiente de trabalho como segunda perspectiva de mensuração no estudo já definido como explanatório sequencial, uma vez que teria afinidade com a questão da satisfação profissional e da própria gestão de recursos humanos e do trabalho em enfermagem.

De posse a tais escolhas e imerso no "universo" da pesquisa mista, iniciou-se o planejamento e o próprio desenho da investigação, demonstrado no Quadro 1, o qual foi estruturado a partir dos elementos do Projeto de Pesquisa, do planejamento de ações e suas justificativas.

\begin{tabular}{|c|c|c|}
\hline $\begin{array}{c}\text { Elementos do Projeto } \\
\text { de Pesquisa }\end{array}$ & Planejamento & Justificativa \\
\hline $\begin{array}{l}\text { Aporte teórico/revisão } \\
\text { de literatura }\end{array}$ & $\begin{array}{l}\text { Gestão da } \begin{array}{c}\text { qualidade } \\
\text { hospitalar. } \\
\text { Acreditação }\end{array} \\
\text { Satisfação } \\
\text { ambiente de trabalho da equipe } \\
\text { de enfermagem, com ênfase na } \\
\text { Acreditação. }\end{array}$ & $\begin{array}{l}\text { Viabilizava o suporte teórico } \\
\text { necessário para a construção do } \\
\text { Projeto de Pesquisa, enfocando-se a } \\
\text { Acreditação como objeto central, mas, } \\
\text { que "advém" da Gestão da Qualidade. } \\
\text { A satisfação profissional e ambiente de } \\
\text { trabalho da equipe de enfermagem são } \\
\text { elementos ou fatores de interesse no } \\
\text { escopo da Acreditação, logo, } \\
\text { necessários de aproximação teórica } \\
\text { prévia. }\end{array}$ \\
\hline $\begin{array}{l}\text { Referencial } \\
\text { metodológico }\end{array}$ & Obra de John W. Creswell. & $\begin{array}{l}\text { Foi apontada como destaque } \\
\text { internacional na pesquisa com } \\
\text { métodos mistos. }\end{array}$ \\
\hline Objeto de estudo & $\begin{array}{l}\text { Acreditação hospitalar no } \\
\text { contexto do trabalho da equipe } \\
\text { de enfermagem. }\end{array}$ & $\begin{array}{l}\text { Possibilitava o seguimento da pesquisa } \\
\text { do curso de Mestrado que apontou } \\
\text { lacunas de pesquisa, além de } \\
\text { predileções autorais. }\end{array}$ \\
\hline
\end{tabular}




\begin{tabular}{|c|c|c|}
\hline Objetivo geral & $\begin{array}{llrr}\text { Analisar a } & \text { influência } & \text { da } \\
\text { Acreditação no trabalho } & \text { da } \\
\text { equipe de } & \text { enfermagem } \\
\text { hospitalar ancorada em } & \text { uma } \\
\text { perspectiva } & \text { mista } & \text { de } \\
\text { investigação. } & & \end{array}$ & $\begin{array}{l}\text { Pela factibilidade e ao problema de } \\
\text { pesquisa. }\end{array}$ \\
\hline estudo & $\begin{array}{l}\text { Estudo transversal, ancorado no } \\
\text { método misto, na estratégia } \\
\text { explanatória sequencial. }\end{array}$ & $\begin{array}{l}\text { Supriria a necessidade prévia de } \\
\text { mensurar os "efeitos" da Acreditação } \\
\text { elencados. Ainda, possibilita maior } \\
\text { compreensão, por meio da } \\
\text { subjetividade secundária, dos } \\
\text { fenômenos. }\end{array}$ \\
\hline Loca & $\begin{array}{l}\text { Unidades de Terapia Intensiva } \\
\text { de um hospital certificado pela } \\
\text { Acreditação nacional e de dois } \\
\text { hospitais não certificados, sendo } \\
\text { um público e um privado. } \\
\text { Definição do hospital certificado } \\
\text { pelo sítio eletrônico online da } \\
\text { Organização Nacional de } \\
\text { Acreditação (ONA), de acordo } \\
\text { com critérios de inclusão pré- } \\
\text { definidos e; das instituições não } \\
\text { certificadas pelo Cadastro } \\
\text { Nacional de Estabelecimentos } \\
\text { de Saúde, norteados por critérios } \\
\text { prévios. }\end{array}$ & $\begin{array}{l}\text { Os setores críticos em pauta foram } \\
\text { eleitos a fim de gerar viabilidade ao } \\
\text { estudo e padronização nas } \\
\text { comparações, pois unidades não } \\
\text { críticas tendem a se diversificar entre } \\
\text { instituições hospitalares. Quanto aos } \\
\text { hospitais, encontrava-se subentendida } \\
\text { a comparação pela presença/ausência } \\
\text { da certificação de Acreditação. A } \\
\text { escolha de dois hospitais (um público e } \\
\text { um privado) a serem comparados pela } \\
\text { instituição certificada foi planejada } \\
\text { para fortalecer as análises. Logo, a } \\
\text { compreensão do fenômeno. }\end{array}$ \\
\hline População & $\begin{array}{l}\text { Trabalhadores de enfermagem } \\
\text { das Unidades de Terapia } \\
\text { Intensiva (UTI) dos três } \\
\text { hospitais. Respeito à critérios } \\
\text { estatísticos de amostragem } \\
\text { representativa estratificada (com } \\
\text { apoio de consultoria estatística) } \\
\text { por "tipo" de UTI (adulto e } \\
\text { infantil) e nível hierárquico da } \\
\text { profissão (enfermeiros } \\
\text { profissionais de nível médio). } \\
\text { Delineamento claro de critérios } \\
\text { de inclusão e exclusão, em } \\
\text { ambas as etapas (QUAN e qual) } \\
\text { do estudo. }\end{array}$ & $\begin{array}{l}\text { Necessário para responder o propósito } \\
\text { do estudo. Em relação aos } \\
\text { procedimentos estatísticos, é uma } \\
\text { premissa básica da estratégia } \\
\text { explanatória sequencial eleita como } \\
\text { desenho de estudo misto. }\end{array}$ \\
\hline $\begin{array}{l}\text { Procedimentos } \\
\text { Instrumentos } \\
\text { Coleta de Dados }\end{array}$ & $\begin{array}{l}\text { Abordagem por conveniência } \\
\text { até atingir o dimensionamento } \\
\text { amostral. Aplicação das versões }\end{array}$ & $\begin{array}{l}\text { O procedimento de abordagem por } \\
\text { conveniência até o preenchimento } \\
\text { amostral não se dissociaria da }\end{array}$ \\
\hline
\end{tabular}




\begin{tabular}{|c|c|c|}
\hline & $\begin{array}{l}\text { brasileiras do Index of Work } \\
\text { Satisfaction e Brazilian Nursing } \\
\text { Work Index }- \text { Revised }^{6} \text { na etapa } \\
\text { QUAN, além de formulário } \\
\text { contendo dados } \\
\text { sociodemográfico e profissionais } \\
\text { dos participantes. Na etapa qual, } \\
\text { entrevistas semiestruturadas, } \\
\text { individuais e áudio-gravadas } \\
\text { com profissionais que tivessem } \\
\text { participado da primeira etapa, a } \\
\text { fim de efetivar a conexão de } \\
\text { dados. }\end{array}$ & $\begin{array}{l}\text { estratégia eleita. Os instrumentos } \\
\text { responderiam às necessidades de } \\
\text { mensuração elencadas, por terem } \\
\text { reconhecimento internacional e } \\
\text { validade nacional. Já a entrevista } \\
\text { semiestruturada com profissionais } \\
\text { previamente integrantes do estudo } \\
\text { reforçaria o procedimento de conexão, } \\
\text { previsto na estratégia explanatória } \\
\text { sequencial e já era de experiência } \\
\text { autoral prévia. }\end{array}$ \\
\hline $\begin{array}{l}\text { Procedimentos de } \\
\text { Ordenamento } \\
\text { Análise de Dados }\end{array}$ & $\begin{array}{l}\text { Respeito ao princípio de } \\
\text { conexão previsto na estratégia } \\
\text { explanatória sequencial: Coleta } \\
\text { QUAN - análise preliminar } \\
\text { QUAN; Coleta qual - Análise } \\
\text { qual; Análise conjunta/final. Na } \\
\text { etapa QUAN, após tabulação, } \\
\text { coadunar aos princípios de } \\
\text { estudos transversais (estatística } \\
\text { descritiva e inferencial/analítica) } \\
\text { robustos com uso de algum } \\
\text { software de amparo, ainda com o } \\
\text { apoio da consultoria estatística } \\
\text { já planejada anteriormente. Para } \\
\text { a etapa qual Discurso do Sujeito } \\
\text { Coletivo (DSC), culminando à } \\
\text { interpretação final/conjunta de } \\
\text { todo o banco de dados. }\end{array}$ & $\begin{array}{l}\text { O respeito ao princípio de conexão } \\
\text { (uma das formas de combinação de } \\
\text { dados) é o que caracteriza o estudo } \\
\text { como misto. As análises de teor } \\
\text { estatístico eram previstas e } \\
\text { obrigatórias na estratégia delineada. } \\
\text { Quanto à escolha do DSC como } \\
\text { técnica de análise qual, esta decisão foi } \\
\text { tomada após contato com uma } \\
\text { pesquisadora do Programa de Pós- } \\
\text { Graduação que sediou o estudo, que } \\
\text { possui vasta experiência em } \\
\text { investigações qualitativas, assim como, } \\
\text { por possibilitar o manejo analítico de } \\
\text { grande volume de dados. }\end{array}$ \\
\hline Aspectos Éticos & $\begin{array}{l}\text { Respeito aos princípios que } \\
\text { regem pesquisas com seres } \\
\text { humanos nas fases (QUAN e } \\
\text { qual) do estudo. }\end{array}$ & Necessário e obrigatório. \\
\hline
\end{tabular}

Quadro 1 - Elementos do projeto de pesquisa com métodos mistos, ações de planejamento e justificativas. Paraná, Brasil, 2015-2017

\section{Execução do Projeto de Pesquisa com Métodos Mistos}

O Projeto de Pesquisa que foi submetido a Exame Geral de Qualificação, em junho de 2016, com posterior encaminhamento às instituições a serem investigadas e, após aceite das mesmas, ao Comitê de Ética em Pesquisa envolvendo seres humanos. 
Com a aprovação ética do Projeto em outubro de 2016, deu-se início aos procedimentos operacionais da pesquisa nos campos de estudo em dezembro do mesmo ano. Todo o processo de coleta de dados, de ambas as fases do estudo, teve duração de sete meses. Isso incluiu o momento de conexão de dados, já que o delineamento escolhido previa intercalar processos de coleta QUAN - análise preliminar QUAN - coleta qual - análise final.

A execução do Projeto de Pesquisa com métodos mistos, combinando suas etapas e aspectos definidores de cada procedimento de pesquisa, estão ilustradas na Figura 1. Na figura, elementos quadrados representam etapas do estudo e elementos ovais, procedimentos e estratégias de pesquisa. Vale lembrar que, nesse processo, sempre que necessária, a consultoria estatística se fez presente.

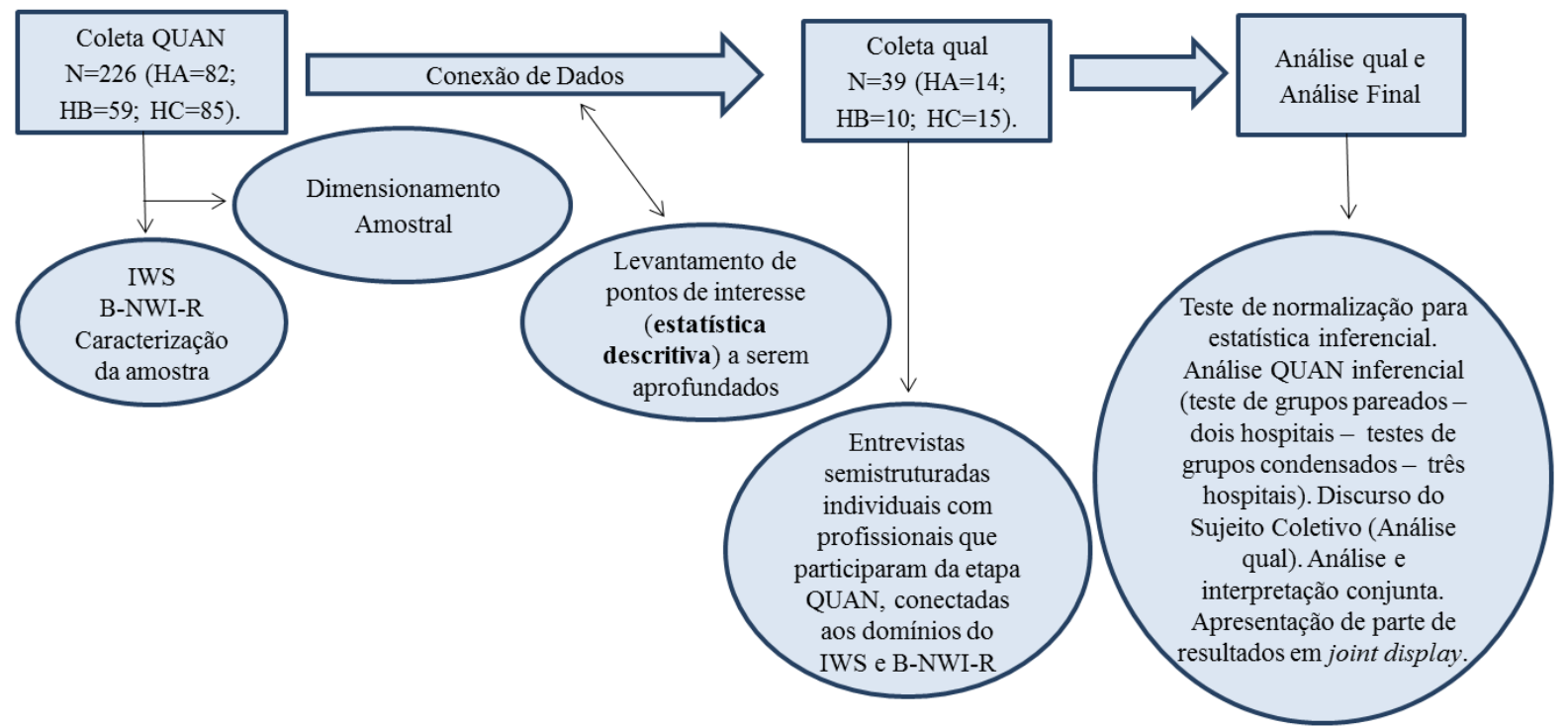

Figura 1 - Esquema de execução do Projeto de Pesquisa com métodos mistos adaptado de Creswell. Paraná, Brasil, 2016-2017

Legendas: QUAN=Quantitativa; qual=Qualitativa; HA=Hospital “A”; HB=Hospital "B”; HC=Hospital "C". IWS=versão brasileira do Index of Work Satisfactiton; B-NWI-R=versão brasileira do Nursing Work Index Revised; joint display=apresentação conjunta de dados quantitativos e qualitativos.

Cada etapa e procedimentos foram executados respeitando-se a estratégia explanatória sequencial das pesquisas com métodos mistos, condizentes com o referencial de Creswell, ${ }^{1,4-5}$ Acerca disso, houve rigor no cumprimento de procedimentos estatísticos, dada à atribuição de 
peso quantitativa da pesquisa, sem, no entanto, esmerar-se no processo de combinação de dados, por meio da conexão entre análise quantitativa preliminar e, coleta de dados qualitativos. Em outras palavras, os dados estatísticos descritivos foram o próprio guia/norte para a coleta de dados qualitativa. Logo, esta teve caráter secundário e de aporte no estudo, mas foi essencial para a melhor/maior compreensão dos fenômenos.

A execução do Projeto de Pesquisa culminou na defesa da Tese de Doutorado ${ }^{12}$ em dezembro de 2017. Após isso, produtos condensados da Tese foram divulgados por meio de publicações. ${ }^{13-14}$ Nestes textos, explicitam-se processos específicos de cada nuance ou "subobjeto" do estudo, quais sejam: o ambiente de trabalho e a satisfação profissional da equipe de enfermagem, no contexto da Acreditação hospitalar.

\section{Considerações finais}

A experiência relatada foi desafiadora e intensa, por demandar dedicação, disciplina e busca constante nos diferentes momentos da pesquisa, aqui descritos como etapas de préconcepção, planejamento e execução do projeto de pesquisa com métodos mistos. Ainda que o processo de delineamento e operacionalização investigativo tenha sido protagonizado por um único pesquisador no desenvolvimento de Tese de Doutorado, houve a clara necessidade de troca de experiências, conhecimento e de apoio técnico-científico em âmbito coletivo.

Destacam-se alguns pontos que podem ser de interesse aos pesquisadores atraídos pelos métodos mistos, principalmente àqueles que se identificarem com a estratégia explanatória sequencial: 1) A consultoria estatística (inclusive anteriormente a fase de coleta de dados) facilitou sobremaneira o cumprimento dos princípios que regem um estudo do tipo survey (transversal); 2) O planejamento de como a conexão de dados seria de fato realizada (entre as fases de análise preliminar de dados QUAN e coleta de dados qual) poderia ter sido melhor elaborado se o pesquisador tivesse previsto mais claramente o volume de dados de estatística 
descritiva. Isso porque, dado ao volume de dados numéricos e a importância do procedimento de conexão no desenho de estudo misto eleito, essa etapa foi consideravelmente atribulada na pesquisa; 3) No que se trata de pesquisas vinculadas à pós-graduação stricto sensu, sugere-se capacitação por meio de disciplina ou curso acerca de métodos mistos e; por fim, 4) É necessária a imersão profunda do pesquisador não somente nos procedimentos metodológicos, como também, na compreensão dos objetos e fenômenos do estudo pois, ao final da pesquisa mista, é provável que o autor se depare com um banco de dados (quantitativos e qualitativos) robusto, que necessariamente deverá ser interpretado de forma conjunta. Portanto, a devida apropriação do próprio objeto de estudo tende a ser um direcionador neste aspecto.

Conclui-se que a experiência em pesquisa com métodos mistos foi profícua. Apesar das barreiras vivenciadas, especialmente no que se refere à viabilidade do estudo em tempo limitado e a abrangência a uma experiência circunscrita, o estudo relatado, possivelmente, contribuiu ao aporte de conhecimento à enfermagem, principalmente por divulgar uma pesquisa de métodos mistos na área de forma processual.

\section{Referências}

1. Creswell JW, Plano Clark VL. Pesquisa de métodos mistos. 2ª ed. Porto Alegre: Penso; 2013.

2. Santos JLG, Erdmann AL, Meirelles BHS, Lanzoni GMM, Cunha VP, Ross R. Integração entre dados quantitativos e qualitativos em uma pesquisa de métodos mistos. Texto \& Contexto Enferm [Internet]. 2017 [acesso em 2019 maio 31];26(3):e1590016. Disponível em: http://www.scielo.br/scielo.php?script=sci_arttext\&pid=S0104-07072017000300330\&lng=en

3. Oliveira JLC, Magalhães AMM, Matsuda LM. Métodos mistos na pesquisa em enfermagem: possibilidades de aplicação à luz de Creswell. Texto \& Contexto Enferm [Internet]. 2018 [acesso em 2019 31 de maio];27(2):e0560017. Disponível em: http://www.scielo.br/scielo.php?script=sci_arttext\&pid=S010407072018000200323\&lng=en

4. Creswell JW. Projeto de pesquisa: métodos qualitativo, quantitativo e misto. $3^{\mathbf{a}}$ ed. Porto Alegre: Artmed; 2010. 
5. Fetters MD, Curry LA, Creswell JW. Achieving integration in mixed methods designs principles and practices. Health Serv Res. 2013;48(6 Pt 2):2134-56. Disponível em: https://www.ncbi.nlm.nih.gov/pmc/articles/PMC4097839/

6. Gasparino RC, Guirardello EB. Translation and cross-cultural adaptation of the "nursing work index" - revised" into Brazilian portuguese. Acta Paul Enferm [Internet]. 2009 [acesso em 2019 jun 04];22(3):2817. Disponível em: http://www.scielo.br/pdf/ape/v22n3/en_a07v22n3.pdf

7. Lino MM. Satisfação profissional entre enfermeiras de UTI: adaptação transcultural do Index of Work Satisfaction (IWS) [dissertação]. São Paulo: Universidade Federal de São Paulo (UNIFESP); 1999. 221 p.

8. Viera CS, Bugs BM, Carvalho ARS, Gaiva MAM, Toso BRGO. Description of the use of integrative mixed method in neonatal nursing. Rev Esc Enferm USP [Internet]. 2019 [acesso em 2019 maio 31];53:e03408. Disponível em: http://www.scielo.br/scielo.php?script=sci_arttext\&pid=S008062342019000100600\&lng=en

9. Santos JLG, Fabrizzio CG, Oliveira JLC, Aires M. Métodos mistos: aspectos operacionais para a pesquisa em enfermagem e saúde. In: Lacerda MR, Ribeiro RP, Costenaro RGS, organizadoras. Metodologia da pesquisa para enfermagem e saúde da teoria à prática. Porto Alegre: Moriá; 2018. p. 20530.

10. Doorenbos AZ. Mixed methods in nursing research: an overview and practical examples.

Kango Kenkyu [Internet]. 2014 [acesso em 2019 maio 31];47(3):207-17. Disponível em: https://www.ncbi.nlm.nih.gov/pmc/articles/PMC4287271/

11. Bressan V, Bagnasco A, Aleo G, Timmins F, Barisone M, Bianchi M, et al. Mixed-methods research in nursing - a critical review. J Clin Nurs [Internet]. 2017 [acesso em 2019 maio 31];26(19-20):2878-90. Disponível em: https://www.ncbi.nlm.nih.gov/pubmed/27805742

12. Oliveira JLC. Influência da acreditação hospitalar no trabalho da equipe de enfermagem: estudo de método misto [tese]. Maringá: Universidade Estadual de Maringá; 2017. 173 p.

13. Oliveira JLC, Souza VS, Pereira ACS, Haddad MCFL, Marcon SS, Matsuda LM. Ambiente de trabalho e acreditação: análise pelo método misto explanatório sequencial. Esc Anna Nery Rev Enferm. 2018 [acesso em 2019 maio 31];22(4):e20170379. Disponível em: http://www.scielo.br/pdf/ean/v22n4/pt_14148145-ean-22-04-e20170379.pdf

14. Oliveira JLC, Magalhães AMM, Bernardes A, Haddad MCFL, Wolff LDG, Marcon SS, et al. Influência da acreditação hospitalar na satisfação profissional da equipe de enfermagem: estudo de método misto. Rev Latinoam Enferm [Internet]. 2019 [acesso em em 2019 maio 31];27:e3109. Disponível em: http://www.scielo.br/pdf/rlae/v27/pt_0104-1169-rlae-27-e3109.pdf 


\section{Autor correspondente}

Nome: João Lucas Campos de Oliveira

E-mail: enfjoaolcampos@yahoo.com.br

Endereço: Rua São Manoel, 963. Santa Cecília. Porto Alegre - RS.

CEP: $90620-110$

\section{Contribuições de Autoria}

1 - João Lucas Campos de Oliveira

Concepção do estudo, coleta dos dados, análise e interpretação dos dados, discussão dos resultados, revisão crítica do conteúdo e aprovação da versão final do artigo.

2 - Ana Maria Müller de Magalhães

Revisão crítica do conteúdo e aprovação da versão final do artigo.

3 - Juliana Aparecida Peixoto Nishiyama

Contribuições: revisão crítica do conteúdo e aprovação da versão final do artigo.

4 - Dagmar Willamowius Vituri

Revisão crítica do conteúdo e aprovação da versão final do artigo.

5 - Sonia Silva Marcon

Revisão crítica do conteúdo e aprovação da versão final do artigo.

6 - Laura Misue Matsuda

Concepção do estudo, revisão crítica do conteúdo e aprovação da versão final do artigo.

\section{Agradecimento}

Ao Conselho Nacional de Desenvolvimento Científico e Tecnológico ( $\mathrm{CNPq}$ ) por fomento de recursos via chamada MCTIC/CNPq № 28/2018

\section{Como citar este artigo}

Oliveira JLC, Magalhães AMM, Nishiyama JAP, Vituri DW, Marcon SS, Matsuda LM. Pesquisa com métodos mistos na enfermagem: experiência na pós-graduação. Rev. Enferm. UFSM. 2019 [Acesso em: Anos Mês Dia];vol9 (Esp): P1-15. DOI:https://doi.org/10.5902/2179769238441 